This document is the accepted manuscript version of the following article:

Schwab, 0. (2019). Construction of ground subtracks for aircraft noise calculations using an estimate of lateral flight dispersion. Acta Acustica United with Acustica, 105(5), 779-783.

https://doi .org/10.3813/AAA. 919358

\title{
Construction of Ground Subtracks for Aircraft Noise Calculations Using an Estimate of Lateral Flight Dispersion
}

\author{
Olivier Schwab ${ }^{1)}$ \\ 1) Empa, Swiss Federal Laboratory for Materials Testing and Research, \\ Laboratory for Acoustics/Noise Control \\ 8600 Dübendorf, Switzerland. olivier.schwab@empa.ch
}

\section{- Summary}

2 In the absence of position data such as radar data, air3 craft noise calculations usually rely on the definition of 4 flight geometries in terms of flight track and flight pro5 file data. Typically, the ground track is constructed as 6 a backbone track with a number of subtracks account7 ing for the lateral flight dispersion. Depending on the 8 nature of the tracks, for instance when considering diverging tracks compared to very narrow tracks, the number of subtracks needs to be such that smooth noise contours and footprints are computed, putting a requirement on the minimum number of subtracks needed. In practice however, as the tracks need to be digitized by expert hand, the number of available subtracks is often limited. Furthermore, the location of the subtracks is often unknown, requiring corridor boundaries to be estimated and to be translated into subtrack locations.

This paper presents a method for the construction of the required number of subtracks based on an estimate of lateral flight dispersion. Two cases are envisioned: the first being an estimate of the lateral flight dispersion using a set of three pre-existing subtracks (one backbone track and left/right subtracks), the second being an estimate using a backbone track and corridor boundaries. The method uses geometric matching of the original tracks, followed by an estimation of the local lateral track dispersion. The lateral distribution function is then used for the construction of new subtracks. A series of aircraft noise calculations using different numbers of subtracks are shown, showcasing the influence of the number of subtracks on the noise contours, depending on the nature of the tracks (e.g. situations with tight turns).

\section{Introduction}

data with the goal of establishing a discrete number of ground tracks, characterized as a backbone track with a number of subtracks representing the lateral dispersion of the flights around the backbone track. General guidelines are provided on which factors should be considered when deriving the ground tracks: the number of subtracks needs to be chosen in such a way that the lateral dispersion is adequately reproduced; the lateral dispersion must be captured and described by a flight distribution leading to the assignment of movements percentages on the subtracks; each route must be given its own set of subtracks, with further aircraft specific subdivisions possible if the data shows considerable differences depending on the aircraft category.

Generally, ECAC Doc. 29 Volume 2 [2] suggests the use of a 7-point approximation (i.e. 1 backbone track with 6 subtracks) and a normal distribution for the description of the lateral distribution, while mentioning that the optimal number of subtracks depends on the situation and needs to be chosen appropriately. The use of a smaller than necessary number of subtracks results in a denting in the noise contours. In practice, radar data is often not available on smaller airfields, and the tracks are drawn by experts using operational knowledge, which introduces limits on the available number of subtracks: an expert is much more likely to identify a backbone track as well as left and right subtracks than to be able to generate a larger number of subtracks that still capture the desired lateral dispersion. In some cases, the flight route can only be characterized in terms of a backbone track and corridor boundaries. This contribution presents a method to construct a required number of subtracks based on an initial input of a backbone and side-tracks, the latter being either a set of pre-existing (but limited in number) subtracks or corridor boundaries for the flight route.

\section{Methods}

The construction of ground subtracks involves two components: first, the geometric matching of the backbone and side tracks (subtracks or corridor 
boundaries), and second, the calculation of the required number of subtracks assuming the same local lateral movement distribution function.

\subsection{Geometric track matching}

For each sample point along the backbone track, the geometric matching assigns corresponding points on each side track. In a first step, all tracks are upsampled as well as smoothed, such that sharp edges in the tracks are attenuated. The track's $X$ and $Y$ coordinates in the horizontal plane are thus available at regular sampling intervals of distance covered along the ground track. Here, a sampling rate of $10 \mathrm{~m}$ is chosen; the smoothing of the tracks is achieved by a moving average over a span of 5 points, applied after the resampling. In addition, the tracks are extended by $10 \mathrm{~km}$ in order to ensure matching backbone and side track points for all track points of the original extend.

The sampled points of the backbone tracks are then matched with those of each side track using dynamic time warping (DTW). DTW is a dynamic programming algorithm that was originally developed for speech recognition [3]. A pair of time-series, that show a similar pattern but are stretched with respect to each other on the time axis, are mapped in time through the use of a warping function. The dynamic programming problem uses a measure of local distance between points on both signals. The sum of these local distance measures is minimized, with a number of boundary conditions (such as restrictions on the warping function or adjustment window conditions), yielding the optimal warping path. The warping path indicates the mapping of the two signals' time axes that gives the best match in terms of similarity in shape 4 .

Here, the method is applied for two-dimensional data series that are sampled along the ground distance. The two-dimensional version of the method (2D-DTW) matches the coordinates $\{X, Y\}$ of the backbone track and the side track,

$$
\left\{X_{B T}, Y_{B T}\right\}\left(s=s_{i}\right) \leftrightarrow\left\{X_{S T}, Y_{S T}\right\}\left(s=s_{j}\right),
$$

where $\left\{X_{B T}, Y_{B T}\right\}$ are the coordinates of the backbone track, $\left\{X_{S T}, Y_{S T}\right\}$ are the coordinates of the subtrack, and $i, j$ are given by the warping path. The coordinates of the backbone track at ground distance $s_{i}$ match the coordinates of the side track at ground distance $s_{j}$. Here, the implementation of [5] is used, which is based on [3, 6].

The sampling rate of both the backbone track and side track is required to be high, because only existing discrete points are matched. The advantage of matching the tracks using DTW, e.g. when compared to building the intercept of the locally perpendicular line to the backbone track sample point with the side track, is that the method prevents returning points in tight corners and issues of sampling for diverging curves, e.g. when the perpendicular line yields no intersection point (Figure 1).

\subsection{Construction of ground subtracks}

After completing the matching (see above), corresponding points on each side track have been determined for each sampled point on the backbone track. In a next step, a connecting line between each pair of matching points is constructed, and the distance between the points is calculated. The distance from the backbone track to the respective side track combined with the movement distribution on the tracks lead to the local lateral distribution function.

First, for the case of the side tracks corresponding to a set of pre-existing subtracks, the standard deviation $\sigma_{k}$ is determined as

$$
\sigma_{k}=\sqrt{\frac{-d_{k}^{2}}{2 \cdot \ln \frac{f_{S T}}{f_{B T}}}},
$$

under the assumption that this distribution is Gaussian, where $d_{k}$ is the distance between the matching points, $f_{S T}$ is the movement percentage of the original subtrack and $f_{B T}$ is the movement percentage of the backbone track. Equation 2 is derived by setting the constraints of $f(d=0)=f_{B T}$ and $f\left(d=d_{k}\right)=f_{S T}$ on the general formulation of the Gaussian distribution. The index $k$ indicates the local match: for each sampled point on the backbone track, there are one or more corresponding points on each subtrack - the distribution is then estimated for those pairs of points, i.e. $\sigma$ can change along the progression of the tracks. If the sampled point on the backbone matches with more than one point on a side track, the estimation is repeated for all pairs of matching points (and thus requires a high sampling rate of the signals). For an initial set of three subtracks, the lateral flight dispersion is characterized by $\sigma_{k}=d_{k} / 1.70$ (with $f_{S T}=16 \%$ and $f_{B T}=68 \%$ ).

Second, for the case of the side tracks corresponding to corridor boundaries, the standard deviation $\sigma_{k}$ is determined as

$$
\sigma_{k}=\frac{d_{k}}{2.5},
$$

where $d_{k}$ is the distance between the matching point and the index $k$ again indicates a local match. Equation 3 reflects the requirement that the subtracks are to be constructed in a swathe of $5 \times \sigma$ as formulated by ECAC Doc. 29 Volume 2 [2.

The processing is done separately for the plane on each side of the backbone track, in order to account for non-symmetrical side track distributions. With the knowledge of the lateral distribution function, a different number of subtracks can be constructed. The spacing between tracks as well as the percentage of 

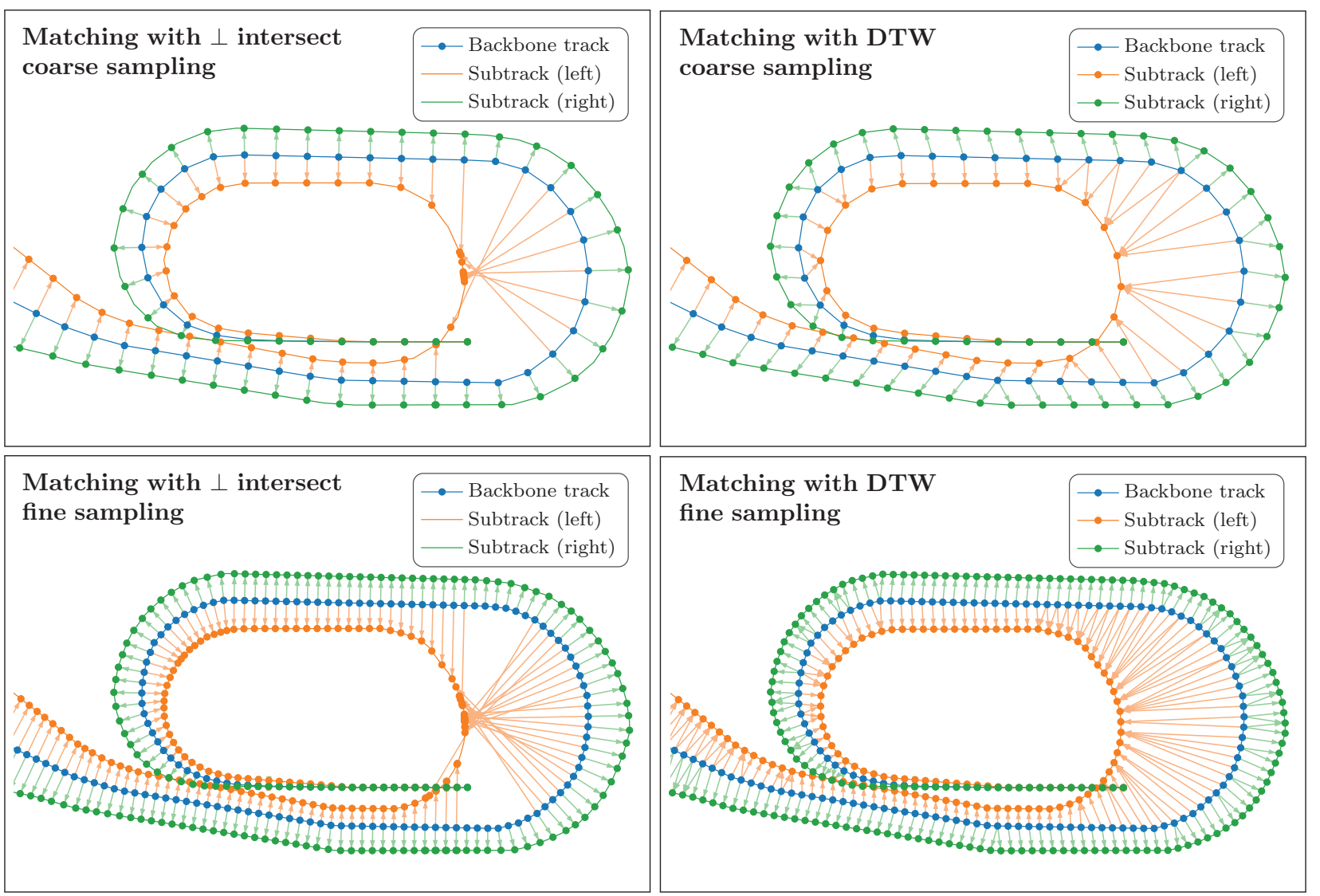

Figure 1: The method using DTW for matching between the tracks is much more robust than a method using matching by perpendicular intersection, provides matches at the sampling rate of the tracks and prevents returning points in tight corners.
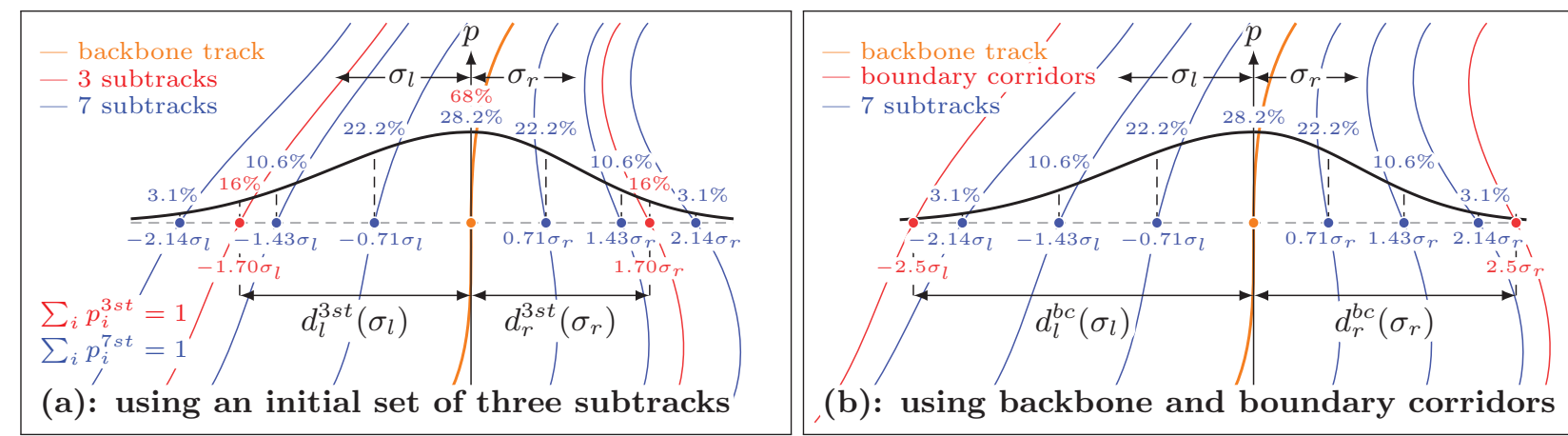

(b): using backbone and boundary corridors

Figure 2: Illustration of the construction process of new subtracks, a) using an initial set of three subtracks and b) using a backbone track and boundary corridors. After the matching of the original tracks, a local distribution function is estimated, which is then used for the construction of the subtracks with the appropriate movement distribution on each track.

movements for 5 to 13 subtracks, under the assumption of a normal distribution with standard deviation $\sigma$, can be found in ECAC Doc. 29 Volume 2, Tables $\mathrm{C}-1$ and $\mathrm{C}-2$ [2].

For each sampled point on the backbone track, the lateral distribution function is estimated. Then, the subtracks are constructed on the connecting line between the matching points, at the distance to the backbone track determined by the distribution as well as the number of subtracks. The process is illustrated by Figure 2 .

As the sampling rate of the curves is required to be high, the resulting tracks are eventually thinned out using the Douglas-Peucker algorithm [7]. The number of points on the track is reduced such that the resulting track stays within a specified tolerance of the initial curve (here, a distance of $10 \mathrm{~m}$ was chosen). This ensures that the number of points is signif- 
icantly reduced for approximately straight segments, but stays high for tight corners and sudden changes in curvatures. In practice, it is also useful to retain some additional points on the runway and at the very end of the tracks, as well as to define a maximal distance between sampling points (e.g. one track point per $500 \mathrm{~m}$ of ground distance). This makes the resulting tracks more robust to further processing, for instance if flight profiles are attached to the track for the generation of complete flight paths. A Matlab implementation of the Douglas-Peucker algorithm for thinning out the track points was taken from the Matlab Central File Exchange 8.

Finally, as the tracks have originally been extended for processing, all tracks are reduced to the original extends. The end point of a newly created subtrack is determined by the intersection between the extended subtrack and the connecting line between the backbone track and the original side track.

\section{Results and discussion}

Results are discussed for three example cases, showcasing tracks with tight turns, non-symmetric subtracks with respect to the backbone track, as well as with different corridor extends. Aircraft noise simulations are done for both a narrow- and wide-body aircraft. All example tracks originate from existing projects, but have been anonymized by translating and mirroring operations as well as simulation on flat terrain. For the purpose of this discussion, the tracks are simulated for A320 and B7773 aircraft, where one example is given for departures and two examples for approaches. Mean altitude and speed profiles for each aircraft type are taken from existing projects, originally derived from radar data. The scenario is fictional, meaning that the altitude profiles are not necessarily appropriate for the given tracks, and the aircraft types that are considered do not necessarily correspond to types for which the tracks were originally created. The simulations are done using FLULA2 Version 004 9.

The original movement distribution is given as $68 \%$ for the backbone track and $16 \%$ for each of the two subtracks. The three tracks (one backbone track and two subtracks) are resampled to seven tracks (one backbone track and six subtracks). The movement percentages and the locations of the new subtracks is given by Tables $\mathrm{C}-1$ and C-2 of ECAC Doc. 29 Volume 2 [2], and determined separately for each side of the backbone track. $28.2 \%$ of the movements are assigned to the backbone track, subtracks $1 / 6$ (left/right) each contain $22.2 \%$ of the movements at the distance $0.71 \times \sigma$ from the backbone track, subtracks $2 / 5$ (left/right) each contain $10.6 \%$ of the movements at the distance $1.43 \times \sigma$ from the backbone track, and subtracks $3 / 4$ (left/right) each contain $3.1 \%$ of the movements at the distance $2.14 \times \sigma$ from the backbone track.

Figure 3 shows the backbone track, the original subtracks as well as the newly constructed subtracks according to the methodology presented in this contribution. In addition, the effect of an increase in the number of subtracks (from three to seven) in the noise $L_{A E}$ footprint is depicted.

Example 1 (Figure 3) shows the difference in the resulting $L_{A E}$ on the receiver grid for an approach 27 , for which the initial subtracks lie far away from each, as well as non-symmetrical subtrack distributions on each side of the backbone track. The inner subtrack is close to the backbone track, and thus increasing the number of subtracks affects the results only little. The outer initial subtrack, in contrast, is so separated from the backbone track that it no longer accurately represents the lateral track dispersion and leads to artifacts in the noise contours. The increase to three subtracks on this side of the backbone track therefore impacts the $L_{A E}$ to up to several $\mathrm{dB}$ around the exposed areas. In this case, an even larger number of subtracks could be appropriate. Alternatively, the route could be split into subroutes, each containing an individual set of subtracks.

Example 2 (Figure 3) shows the difference in the resulting $L_{A E}$ on the receiver grid for an approach 09, for which the initial subtracks follow the backbone track close to the airport, but deviate further at larger distances from the airport. In this case, the differences in the $L_{A E}$ are restricted to the areas directly under the flight path, and become more pronounced as the subtracks deviate further away. In this case, a larger number of subtracks should be considered if the areas farther away from the airport are important for the noise contours.

Example 3 (Figure 3) shows the difference in the resulting $L_{A E}$ on the receiver grid for a departure 27 , for which the initial subtracks follow the backbone track closely, both in close vicinity and farther away from the airport. In this case, the differences in the $L_{A E}$ are negligible, and the choice of three subtracks would be sufficient to represent the lateral track distribution.

\section{Conclusion and outlook}

This paper presented a method for creating ground subtracks based on an initial estimate of lateral flight dispersion, for instance for increasing the number of subtracks based on an original set of a given number of tracks. The method successfully handles tracks with tight turns, widely dispersed tracks as well as non-symmetric track distributions, and does not require meticulous fine-tuning of the sampling rate of the tracks. A number of examples were presented, showcasing the influence of an increased number of subtracks on aircraft noise calculation results. As expected, differences in the calculated sound exposure 

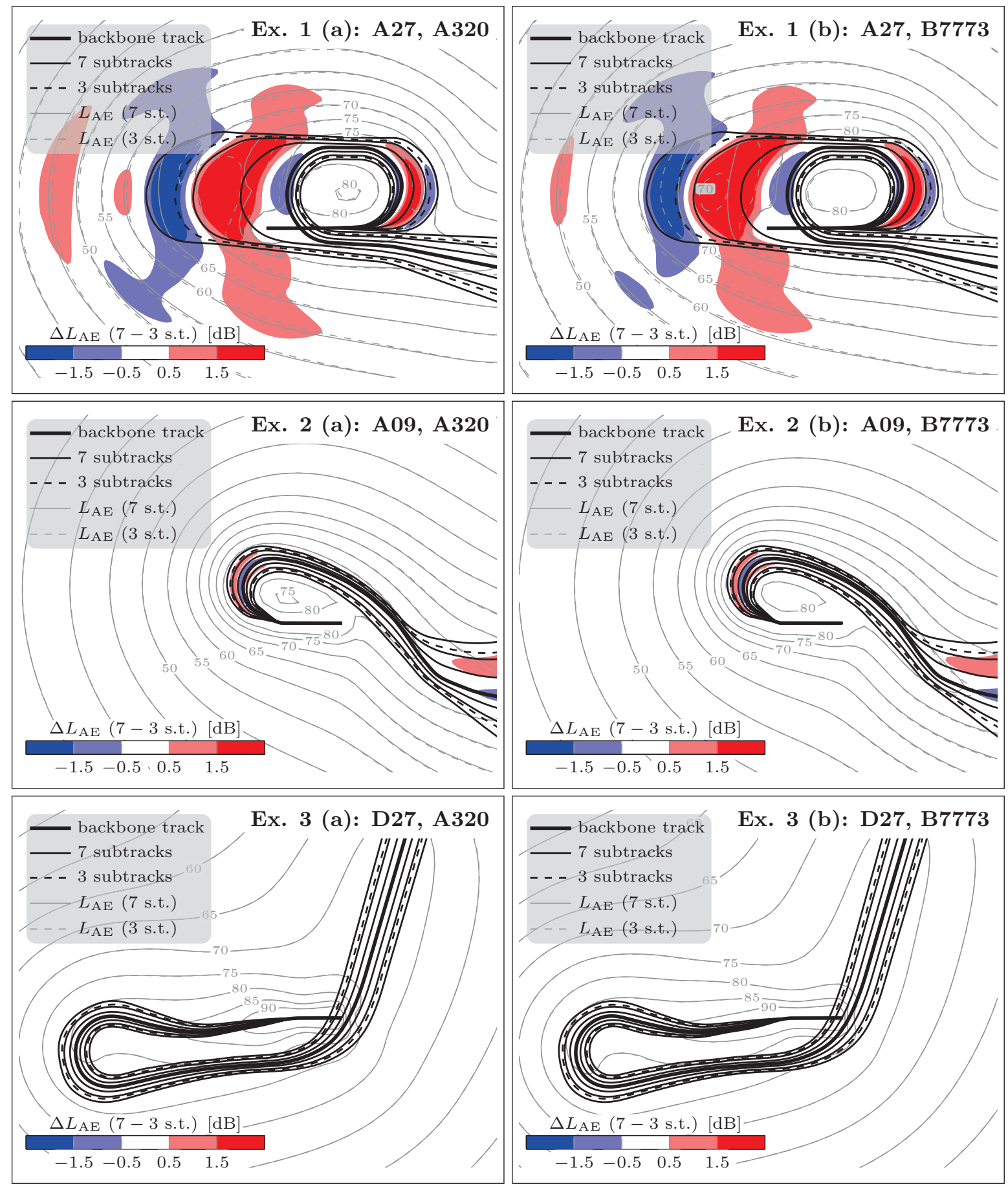

Figure 3: Differences in the resulting $L_{A E}$ on the receiver grid between the calculation using 7 and 3 tracks for two approaches (example 1 and example 2) and one departure (example 3), for A320 and B7773 aircraft.

occur for geometries with large dispersions that cannot be accurately represented by a small number of subtracks, while for flight routes with small lateral dispersion, a reduced number of subtracks can be sufficient.

In this contribution, the transition from three to seven tracks, assuming a normal lateral flight distribution was shown. With slight modifications, the method is applicable to an arbitrary number of tracks without the assumption of a normal distribution. The first step is identical to the process shown here: for each point on the backbone track, the matching points on the subtracks are determined using 2D-DTW. Then, a curve is fitted through the matching points, starting from the point on the backbone track. This curve, which, in general, is not restricted to be linear, allows the estimation of the probability density function as a function of the distance between the point 
on the backbone track and the matching points on the subtracks along the fitted curve. The distribution does not need to be normal, but it is required to be monotonously decreasing (otherwise, no unique solutions can be determined during the construction of the new subtracks). Using the number of desired subtracks and the movement distributions on these new tracks, the probability density function is then used to calculate the distance corresponding to each probability alongside the fitted curve of the matching original points. The new subtrack points are determined as the points at the calculated distances on the curve.

In a further step, it could be envisioned to determine criteria in order to automatically identify the minimum number of required subtracks for a given geometry, e.g. with a criterion based on the geometric distance between tracks.

\section{References}

[1] B. Schäffer, R. Bütikofer, S. Plüss, and G. Thomann. Aircraft noise: accounting for changes in air traffic with time of day. The Journal of the Acoustical Society of America, 129(1):185199, 2011.

[2] ECAC. Doc 29 4th edition, Report on Standard Method of Computing Noise Contours around Civil Airports, Volume 2: Technical Guide. Technical report, European Civil Aviation Conference (ECAC), Neuilly-sur-Seine, France, 2016.

[3] H. Sakoe, S. Chiba, A. Waibel, and K.F. Lee. Dynamic programming algorithm optimization for spoken word recognition. Readings in speech recognition, 159:224, 1990.

[4] M. Müller. Dynamic time warping. Information retrieval for music and motion, pages 69-84, 2007.

[5] P.I. Nerseth. FindSimilar Audio Search Utility utilising MFCC methods. Github repository: https://github.com/perivar/FindSimilar. 2014.

[6] K.K. Paliwal, A. Agarwal, and S.S. Sinha. A modification over Sakoe and Chiba's dynamic time warping algorithm for isolated word recognition. Signal Processing, 4(4):329-333, 1982.

[7] D.H. Douglas and T.K. Peucker. Algorithms for the reduction of the number of points required to represent a digitized line or its caricature. Cartographica: the international journal for geographic information and geovisualization, 10(2):112-122, 1973.

[8] W. Schwanghart.

Line Simplification.
MATLAB Central File Exchange: https: 2010.

[9] Empa. FLULA2 - Ein Verfahren zur Berechnung und Darstellung der Fluglärmbelastung. Technical report, Empa, Swiss Federal Laboratories for Material Science and Technology, Laboratory for Acoustics/Noise Control, Dübendorf, Switzerland, 2010. 